\title{
Efecto de la combinación metodológica de aula invertida e instrucción entre pares en las calificaciones académicas de estudiantes universitarios de Ciencias de la Actividad Física y del Deporte \\ Effect of the methodological combination based on flipped classroom and peer instruction on the grades of Physical Activity and Sport Sciences university students

\author{
D avid Falcón M iguel, Javier Sevil-Serrano, Carlos Peñarrubia-Lozano, Ángel Abós
} \\ Universidad de Zaragoza (España)
}

\begin{abstract}
Resumen: El desar rollo de nuevos métodos de enseñanza-aprendizaje en la educación superior demanda la aportación de evidencias científicas que señalen su efectividad en el rendimiento académico del alumnado. El presente estudio evalúa el efecto de un programa de intervención basado en la combinación de las metodologías de aula invertida e instrucción entre pares sobre una parte de las cal ificaciones académicas obteni das por estudiantes universitarios en una asignatura. Se real izó un diseño cuasi-experimental en el que participaron 52 estudiantes universitarios del $3^{\circ}$ curso de Grado en Ciencias de la Actividad Físicay el D eporte que cursaban la asignaturade Gestión y 0 rganización de Eventos. El grupo experimental recibió durante tres clases los contenidos de la asignatura a través de la combinación metodológica de aula invertida y de instrucción entre pares, mientras que el grupo control lo hizo siguiendo una metodología tradicional. Para comparar la eficacia de la intervención, se midió la calificación académica de un contenido de la asignaturaa través de un examen teórico. Los resultados del estudio mostraron una asociación positiva y significativa entre recibir clases con la combinación metodológica y mayor acierto en las preguntas del examen. Por tanto, la combinación metodologica utilizada parece mostrarse más eficaz que la metodología tradicional para mejorar la compresión de conceptos teóricos en el alumnado universitario.
\end{abstract}

Palabras clave: aula invertida; rendimiento académico; universitarios; instrucción entre pares.

\begin{abstract}
The development of new teaching-learning methods in higher education require the contribution of scientific evidence that indicates whether these types of methodologies are effective. The aim of this study was to assess the effect of an intervention programme based on the combination of flipped classroom and peer instruction on the grades of university students in an academic subject. Fifty-two university students of the $3^{\text {rd }}$ course of Physical Activity and Sports Sciences Degree participated in thisquasi-experimental study. The contents of the subject weretaught through three lessons by a methodological combination of flipped classroom and peer instruction in the experimental group, while in the control group the same contents and number of lessons were taught following a traditional methodology. The students' grades were measured in a part of the subject by means a theoretical exam to assess the effectiveness of the two types of methodologies implemented. The results of the study showed a positive and significant association between receiving lessons with the methodological combination and getting the different exam questions right. Therefore, the combination of this methodologies seems to be more effective than the traditional methodology for improving the understanding in university students.
\end{abstract}

Keywords: flipped classroom; academic achievement; undergraduates; peer instruction; assessment.

\section{Introducción}

La educación superior está sufriendo rápidas transformaciones que suponen un cambio profundo en los procesos de enseñanza y aprendizaje (Hincapie-Parra, Ramos-M onobe \& Chirino-Barceló, 2017). La nueva estructura curricular, el desarrollo de nuevos métodos de enseñanza aprendizaje (e.g, aula invertida o flipped classroom, gamificación, etc.) y la nueva concepción del

Fecha recepción: 06-10-20. Fecha de aceptación: 27-11-20 David Falcón Miguel

dfalcon@unizar.es trabajo del profesorado están generando unas exigencias pedagógico-didácticas sin precedentes en las universidades europeas (Hinojo-Lucena, Aznar-Díaz, Romero-Rodríguez \& Marín-M arín, 2019). Bajo este prisma, en la actualidad, se está vislumbrando un cambio en el rol docente adoptado en las clases. A grandes rasgos, se está pasando del profesorado universitario que ejerce una transmisión unidireccional de conceptos y procedimientos imponiendo un rol pasivo a sus estudiantes (i.e. instrucción directa), a un profesorado que actúa como guía y mediador del aprendizaje y que sitúa al alumnado en el centro del proceso de enseñanza-aprendizaje, organizando experiencias significativas y super- 
visando sus tareas de estudio (Hinojo-Lucena et al., 2019). Además, este cambio de enfoque docente universitario está en línea con la estructura de tiempo que plantea el Sistema Europeo de Transferencia y Acumulación de Créditos (ECTS; European Credit Transfer and Accumulation System) de los nuevos grados universitarios, donde el $66 \%$ del mismo está dedicado al estudio autónomo, mientras que el 33\% restante está destinado a actividades presenciales en el aula que promuevan la reflexión y el análisis crítico (De Miguel, 2006).

Diferentes estudios señalan que los estudiantes se sitúan bajo diversos enfoques, los cuales están ubicados a lo largo de un continuo desde los más superficiales a los más profundos, para enfrentarse y desarrollar sus tareas de aprendizaje (e.g., Biggs, 2005; Kember, Wong \& Leung, 1999). Estos enfoques, están en línea de la taxonomíade Bloom (Figura 1), en la que queda recogidaestaevolución en el proceso de aprendizaje(Anderson $\&$ K rathwohl, 2001). Por un lado, en el enfoque superficial, el alumnado manifiesta la intención de superar la tarea de aprendizaje con el mínimo esfuerzo. Este enfoque, va asociado a un concepto pasivo del aprendizaje, donde los estudiantes se centran en elementos externos del contenido, realizando actividades de un bajo nivel cognitivo y con escasa comprensión (Berbén, Pichardo $\&$ de la Fuente, 2007). En el polo opuesto, el enfoque profundo exige a los estudiantes un abordaje de las ta reas de aprendizaje de un modo global. En este enfoque el alumnado se implica en el proceso adquiriendo un rol activo, relacionando conocimientos previos y nuevos aprendizajes y trabajando por lograr una integración entre la teoría y la práctica (Berbén et al., 2007). En este punto, parece importante señalar que el aprendizaje, sea superficial o profundo, no depende tanto de aquellos conceptos que se imparten, sino de la metodología docente utilizada para su asimilación (López-Pastor, M olina-Soria, Pascual-Arias, \& M anrique Arribas, 2020; Sevil-Serrano, Aibar-Solana, Abós \& García-

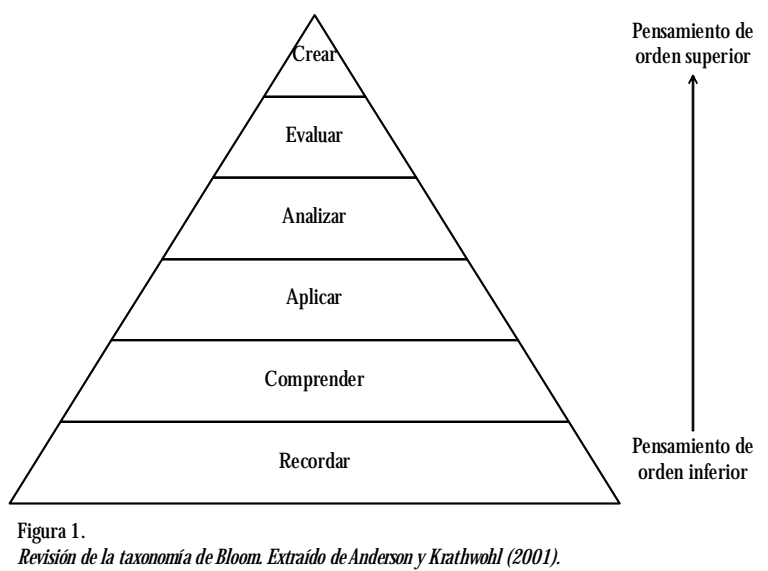

González, 2017). Así, dos metodologías que han mostrado resultados beneficiosos en el aprendizaje en los últimos años en diferentes contextos, debido al rol activo que otorgan al alumnado, son el aula invertida y la instrucción entre pares (Hernández-Campos \& M urilloQ uirós, 2019; Hinojo-Lucena et al., 2019; Recio-Moreno, Feliz-M urias \& Elorza-Barbajero, 2021).

Por un lado, el aula invertida, también denominada clase inversa o modelo invertido de aprendizaje, es una metodología de enseñanza en la que el alumnado adquiere un rol más activo y participativo en su propio proceso de aprendizaje (Hinojo-Lucena et al., 2019; Cuenca-Ruano, García-Martínez, Ferriz-Valero \& Tortosa-Martinez, 2021). La finalidad última de esta metodología radica en la optimización del tiempo de aula para favorecer la comprensión y aplicabilidad de loscontenidosdelaasignatura(Arráez, Lorenzo, Gómez $\&$ Lorenzo, 2018). Para ello, el docente debe facilitar el material relacionado con la materia que, posteriormente, seráanal izado en el aula. Paralelamente, el alumnado debe realizar un trabajo autónomo fuera del aula, revisando los conceptos y contenidos teóricos facilitados por el docente, paradialogar críticamentesu aplicación práctica en el aula (Sola, Aznar, Romero \& RodríguezGarcía, 2019). La metodología de aula invertida puede complementarse con la metodología de enseñanza justo a tiempo (just on time teaching), la cual se basa en la realización de tareas previas a la clase tales como la visualización de un video o la lectura de un artículo, complementadas por preguntas de autoevaluación ( $\mathrm{Hew}$ \& Lo 2018). De este modo, en la metodología de aula invertida las habilidades de orden inferior que aparecían anteriormente recogidas en la taxonomía de Bloom (i.e., memorizar y comprender) se destinan al trabajo autónomo del alumnado fuera del aula, mientras las ha bilidades de orden superior (i.e., aplicar, analizar, evaluar y crear) se abordan en el aula de manera colaborativa entre el alumnado y el profesorado (Parra, 2017).

$S i$ bien algunos estudios señalan que el profesorado puede mostrarse reticente a aplicar esta metodología debido al alto tiempo de preparación de los materiales (Andrade \& Chacón, 2018), diferentes revisiones sistemáticas y metaranálisis, realizados en una gran variedad de disciplinas académicas en contextos universitarios, han reportado mejores calificaciones académicas en el alumnado que recibió en una metodología de aula invertida frente aquellos que recibieron una metodología tradicional (Chen et al., 2018; Hew \& Lo 2018; Hinojo-Lucena et al. , 2019; Sola et al. , 2019). Algunas de las razones que pueden explicar este hecho son el aumento 
de la motivación de los estudiantes hacia la materia debido a la forma de abordar los contenidos en las sesiones o la posibilidad de comprobar el nivel de conocimiento y comprensión de forma previa al examen (i.e., autorregulación) (Hinojo-Lucena et al., 2019). No obstante, aunque menos numerosos, también existen algunos estudios que no han reportado diferencias en el rendimiento académico después de la aplicación de unametodología de aula invertida (e.g., Wanner $\&$ Palmer, 2015; W ilcox, 2014). De este modo, parecen necesarias más investigaciones para encontrar una evidencia concluyente sobre la aplicación de dicha metodología.

Por otro lado, la instrucción entre pares consiste en introducir durante la clase expositiva preguntas conceptuales de respuesta estructurada, que pueden ser presentadas en formato de respuesta dicotómica o de opción múltiple(Maldonado-Fuentes \& Rodríguez-Alveal, 2016). Dichas preguntas están relacionadas con lecturas previas a la clase, las cuales también pueden formar parte de la metodología de enseñanza justo a tiempo (Balta, Michinov, Balyimez \&Ayaz, 2017). Existeun gran abanico de aplicaciones educativas (e.g., Google Forms, Formdesk, etc.) utilizadas para que el alumnado responda a una serie de preguntas de comprensión conceptual sobre dichos materiales proporcionados. Posteriormente, a partir de la revisión de las respuestas, se reorientan los contenidos de la clase y se profundiza en los mismos a través de nuevas tareas de aprendizaje (Hernández-Campos \& M urillo-Q uirós, 2019). Así, la aplicación de la instrucción entre pares en una sesión presencial se desarrolla siguiendo los pasos que muestra la Figura 2.

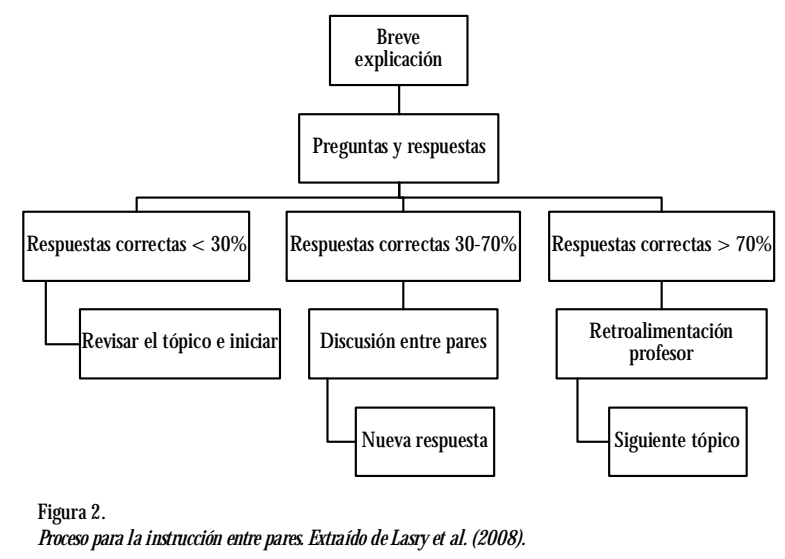

En primer lugar, el docente realiza la presentación del contenido a tratar de manera introductoria, para, seguidamente, pasar a una fase donde aparecen los test conceptuales (Lasry, M azur, \& Watkins, 2008). El estudiante tiene dos oportunidades para responder las pre- guntas. Durante la primera ocasión, dispone de aproximadamente un minuto para contestar de manera individual y en silencio. En el segundo paso, debe discutir la respuesta previamente con algún compañero. Esta parte es la que caracteriza a la metodología, ya que el alumnado interactúa, comenta y dialoga de manera crítica con el resto de sus compañeros (Lasry et al., 2008). Al mismo tiempo, el docente va prestando atención a los argumentos de los estudiantes para conocer el grado de asimilación de los contenidos impartidos. Al final de la sesión, el profesorado revela la solución del ejercicio planteado (Maldonado-Fuentes \& Rodríguez-Alveal, 2016). Para la aplicación de esta metodología, se calcula un tiempo aproximado de 10 a 20 minutos, en una clase teórica de 60 minutos (i.e., un tercio de la sesión). No obstante, este tiempo puede oscilar en función de la cantidad de preguntas, del nivel de acierto en las respuestas, del tema 0 de la ratio de estudiantes, entre otrosfactores(M aldonado-Fuentes \& Rodríguez-Alveal, 2016). Este cambio en la metodología alejada de la clá sica la instrucción directa, además de facilitar la participación de los estudiantes, permite identificar dificultades en la comprensión de los conceptos claves (Lago et al., 2012).

En este sentido, estudios previos han señalado que la metodología de la instrucción entre pares puede mejorar el nivel de aprendizaje, la motivación y el rendimiento académico tras discutir las respuestas con otros estudiantes (e.g., Balta et al., 2017; Hernández-Campos \& Murillo-Quirós, 2019; Vickrey, Rosploch, Rahmanian, Pilarz \& Stains, 2015). Por ejemplo, estudiantes de la U niversidad de Harvard que recibieron una serie de contenidos a través de una metodología basada en la instrucción entre iguales durante un semestre obtuvieron valores significativamente superiores en la comprensión conceptual respecto a otros estudiantes que recibieron esos mismos contenidos a través de la instrucción directa (Lasry et al., 2008). Igualmente, otro estudio más reciente en estudiantes de Física, demostró que aquellos que recibieron una metodología de instrucción entre pares y enseñanza justo a tiempo durante 32 sesiones de una hora, obtuvieron calificaciones académicas más altas respecto a otros estudiantes que recibieron los contenidos mediante instrucción directa(Hernández-Campos\& M urillo-Q uirós, 2019). De este modo, parece que la terminología y el vocabulario utilizado entre los estudiantes a la hora de expresarse y cooperar podría facilitar una mejor comunicación entre pares y, por ende, mejorar la comprensión de los conceptos (Bermejo-Díaz, Pulido-Salas, Galmés-Panadés, 
Serra-Payeras, Vidal-Conti \& Ponseti-Verdaguer, 2021).

Aunque existe un gran número de estudios que ha examinado los efectos independientes de la metodología aula invertida y de instrucción entre pares en el rendimiento académico (e.g., Hernández-Campos \& Murillo-Q uirós, 2019; Hinojo-Lucena et al., 2019; Sola et al., 2019), paraconocimiento de los autores, no existen estudios que hayan examinado los efectos de la combinación de estas dos metodologías en las calificaciones académicas de estudiantes universitarios. Asimismo, debido a la idiosincrasia del Grado en Ciencias de la Actividad Física y el Deporte, con una carga lectiva con un alto componente práctico, el número de estudios realizados en este Grado es muy limitado (Zainuddin \& Halili, 2016). De esta forma, parecen justificados los estudios que ahonden en las posibles bondades de la hibridación de ambas metodologías (i.e., aula invertida y de instrucción entre pares) en estudiantes universitarios de Ciencias del Actividad Física y el Deporte. Con el objetivo de superar esta brecha de estudio, la presente investigación pretende evaluar el efecto de un programa de intervención basado en la combinación de las metodologías de aula invertida e instrucción entre pares sobre las cal ificaciones académicas obtenidas por los estudiantes universitarios en una asignatura de grado en Ciencias de la Actividad Física y del Deporte. En línea con investigaciones previas en estudiantes universita rios llevadas a cabo independientemente con la metodología de aula invertida (e.g., Hinojo-Lucena et al., 2019; Sola et al., 2019) o de instrucción entre pares (e.g., Hernández-Campos \& M urillo-Quirós, 2019; Lasry et al., 2008), se espera que el alumnado que reciba una combinación de ambas metodologías (i.e., grupo experimental), en comparación con el alumnado que reciba clases magistrales con instrucción directa (i.e., grupo control), obtenga un aprendizaje más significativo reflejado en reportar mayores tasas de acierto en las respuestas al examen teórico de la asignatura en la que se llevó a cabo el estudio.

\section{M étodo}

\section{Diseño}

Se realizó un diseño cuasi experimental con grupo control (Thomas, Nelson \& Silverman, 2011) en estudiantes pertenecientes a la asignatura de Gestión y O rganización de Eventos del Grado en Ciencias de la Actividad Física y del Deporte. Los criterios de inclusión para la selección del alumnado fueron la asistencia a las tres clases presenciales impartidas, tanto con la metodología de aula invertida e instrucción entre pares (i.e., grupo experimental), como con la metodología de enseñanza tradicional (i.e., grupo control), así como la realización del examen en la primera convocatoria de la asignatura (i.e., en ambos grupos).

\section{Participantes}

De una muestra inicial de 86 estudiantes de 30 del Grado de Ciencias de laActividad Física y el Deporte, después de aplicar los criterios de inclusión menciona dos, finalmente participaron en el estudio 52 (M edad = 22.09; DT $=3.03,79 \%$ hombres). El grupo experimental estaba formado por 22 estudiantes ( $M$ edad $=$ 22.10; DT = 3.78, 81\% hombres) delapromoción 20182019, quienes tenían una nota media del expediente de 6.8/ 10. El grupo control estaba compuesto por 30 estudiantes ( Medad $=22.71 ;$ DT $=2.28,76 \%$ hombres) de la promoción 2017-2018. Lanota media del expediente del grupo control era de 6.4/ 10. Todos ellos, estaban matriculados en la misma U niversidad Pública, ubicada en una ciudad de tamaño medio del noreste de España.

\section{Instrumentos}

Para evaluar las calificaciones académicas de los estudiantes se formularon cuatro casos relacionados con los elementos básicos relacionados con el aseguramiento en eventos deportivos. En cada uno de ellos, se presentaba una situación hipotética donde había tenido lugar un accidente durante el transcurso de una actividad deportiva. Tras la descripción de la situación, se planteaban dos preguntas: una primera en la que había que responder con el tipo de póliza del seguro que cubría el siniestro (i.e., responsabilidad civil 0 accidentes) y una segunda donde era necesario identificar al tomador de la póliza en cuestión (i.e., participante, organizador 0 titular de la instal ación). Cadauna de las respuestas acertadas tenía asignadauna puntuación de 0.25 puntos en el examen final, lo que implica un valor de 0.5 puntos por

\footnotetext{
Tabla. 1.

№ de Versión reducida de la descripción del caso Póliza Tomador

Un jugador de bádminton se desplaza a gol pear un volante y golpea a $R C^{*}$ Part. 1 un espectador que está junto a la zona de juego provocándole con su $A C$ Org.* raqueta un corte.

A un jugador de pádel se le escapa la pala realizando una acción de RC Part. juego y golpea a su compañero provocándole un corte.

Nota: RC: Responsabilidad Civil; AC: Accidentes; Part: Participante; Org: Organizador; PI:

Propietario o titular de la instalación. Las respuestas correctas han sido identificadas con*.
} 
caso y 2 puntos sobre 10 en la calificación del examen (i.e., 20\% de la calificación final). Los cuatro casos que se presentaron tanto en el examen del grupo control como del grupo experimental quedan recogidos de forma resumida en laTabla 1.

\section{Procedimiento}

El presente estudio siguió las directrices éticas generales de la Declaración de Helsinki (2013) respecto a la interacción con seres humanos, consentimiento y confidencialidad de las respuestas. Asimismo, se contó con el dictamen ético favorable para su desarrollo de la Universidad de los autores. Tanto en el grupo experimental como en el grupo control se impartieron tres clases de 90 minutos de duración. El mismo docente impartió las tres clases en el grupo experimental y control en diferentes cursos académicos. Durante el curso 2017/ 2018, en el grupo control se impartieron los contenidos a través de una metodología tradicional. Durante el curso 2018/ 2019, en el grupo experimental se impartieron los mismos contenidos del curso anterior a través de la combinación metodológica de aula invertida e instrucción entre pares. No hubo modificaciones respecto a la guía docente de referencia entre los dos cursos académicos anal izados. Las preguntas de evalua ción en el examen teórico fueron las mismas para ambos grupos. De este modo, la metodología de enseñanza (i.e., variable independiente) fue el único aspecto que se modificó en los dos cursos académicos.

\section{Programa de intervención}

El programa de intervención aplicado en el grupo experimental estuvo formado por tres sesiones. En la primera sesión se trataron los conceptos básicos de referencia para la contratación de seguros (i.e., seguro, prima, tomador, siniestro e indemnización). En la segunda sesión se atendió a las particularidades de los seguros de responsabilidad civil y de accidentes. Finalmente, en la tercera sesión se estudiaron los elementos del triángulo de seguridad (i.e., instalación, organiza dor y participante) y se analizaron al gunos casos prácticos.

En el grupo experimental se implementó una metodología basada en la combinación metodológica entre aula invertida y la instrucción entre pares. Para ello, previamente al desarrollo de cada una de las sesiones, el alumnado tenía que buscar información, ver unos vídeos y leer unos materiales curriculares. Como guía y facilitador del proceso, el docente subió a la plataforma M oodle (utilizada como herramienta de seguimien- to de la asignatura y comunicación entre el alumnado y el profesorado) materiales e información sobre los conceptos a desarrollar durante las dos primeras sesiones. En la tercera sesión, en el documento específico de la sesión se añadió un enlace a un vídeo donde se exponían diversos casos prácticos de posibles accidentes, con la resolución sobre la culpabilidad y responsabilidad de los siniestros mostrados.

Durante el desarrollo de las sesiones presenciales en clase, el grupo experimental siguió una metodología de instrucción entre pares a través de la aplicación informática Kahoot (Bicen \& Kocakoyun, 2018). Esta herramienta permite elaborar cuestionarios de opción múltiple, que pueden ser resueltos por los estudiantes presencialmente en la clase con sus móviles u ordena dores, permitiendo obtener un feedback inmediato tanto al alumnado como al profesorado. Concretamente, tras una breve explicación, el profesor formulaba una preguntade opción múltiple sobre los contenidos de la asignatura. Cada estudiante disponía de dos de minutos para seleccionar su respuesta de manera individual y enviarla al profesor. En función del porcentaje de acierto obtenido en dicha pregunta, el profesorado reorientaba el proceso de enseñanza-aprendizaje siguiendo las indicaciones sugeridas por Lasry et al. (2008). Si el porcenta je de acierto en una pregunta era inferior al $30 \%$, el profesor ofrecía una nueva explicación con detalles adicionales antes de volver a realizar nuevamente la pregunta. En esta segunda oportunidad, la respuesta se debía discutir previamente en grupos reducidos (formados por entre dosy cuatro estudiantes) en lugar de individualmente. Si el porcentaje de acierto en una preguntaba se situaba entre el 30 y el $70 \%$, los estudiantes discutían su respuesta con el compañero de al lado durante un máximo de cuatro minutos, dial ogando y argumentando su razonamiento. Durante este tiempo, el profesor recorría el aula escuchando y guiando a los estudiantes en su propio razonamiento. Transcurrido el tiempo dado, el profesor solicitaba las respuestas fina les, obteniendo un feedback del nivel de comprensión del tema por parte de los estudiantes. En el siguiente paso, el profesor ofrecía una retroalimentación a todo el grupo sobre la respuesta correcta y su justificación. Finalmente, si el porcentaje de acierto en una pregunta superaba el $70 \%$, el docente justificaba la respuesta y se pasaba a la siguiente.

\section{Análisis de datos}

Todos los datos del presente estudio fueron tratados mediante el software estadístico IBM SPSS 21.0. De 
forma previa, se comprobó la homogeneidad entre los gruposexperimental y control en cuanto al género, edad y expediente académico, utilizando para ello las pruebas chi-cuadrado y t-student. Para examinar si la intervención llevada a cabo había sido efectiva se examina ron las diferencias en las calificaciones académicas entre el grupo experimental y el grupo control a través de tablas de contingencia cruzadas. Para ello, se utiliza ron los estadísticos chi-cuadrado y V de Cramer. Los valores deV de Cramer superiores a .10, a .30 y a .50 fueron considerados pequeños, moderados y grandes, respectivamente (Cohen, 1988). Respecto al análisis de los residuos ajustados (ra), se tomó un valor mayor que dos (i.e., ra> 2) o menor que menos dos (i.e., ra< «2) para establecer una relación significativa, excitadora 0 inhibitoria, respectivamente. El nivel de significación estadística considerado fue $p<.05$.

\section{Resultados}

De forma previa a la realización de los análisis principales del estudio, se analizaron las posibles diferencias entre los grupos experimental y control en cuanto al género, la edad y la nota de acceso a la universidad. En primer lugar, se realizó una prueba chi-cuadrado que determinó que el grupo experimental y el grupo control no presentaban diferencias en cuanto su composición por género $\left(x^{2}(1)=0.17 ; V=.05 ; p>.05\right)$. Seguidamente, realizó una prueba t-student, la cual determinó que no existían diferencias entre el grupo experimental y control ni en relación a la edad $(t=1.21$; $p>.05$ ), ni en relación a la nota media del expediente ( $t$ $=1.92 ; p>.05)$. En su conjunto, estos hallazgos determinaron que ambos grupos eran homogéneos.

Posteriormente, para comprobar si la intervención había sido efectiva se examinaron las diferencias en las calificaciones académicas entre el grupo experimental y el grupo control. La relación entre haber recibido las tresclases con unametodología tradicional o con la combinación metodológica de aula invertida e instrucción entre pares y haber respondido correctamente 0 erróneamente a las ocho preguntas planteadas se presenta en laTabla2. En cinco delas ocho preguntas (i.e., 62.5\%; $1,3,5,7$ y 8 ), los resultados mostraron una asociación positiva y significativa entre recibir clases con una combinación metodológica de aula invertida e instrucción entre pares y acertar las diferentes preguntas. De manera opuesta, se apreció una asociación positiva y significativa en cinco de las ocho preguntas (i.e., 62.5\%; 1, $3,5,7$ y 8 ) entre recibir clase con un estilo de enseñan- za tradicional y responder erróneamente las diferentes preguntas. En las otras tres preguntas $(2,4$ y 6$)$ no existió asociación entre el tipo de metodología y contestar acertada 0 erróneamente a las preguntas.

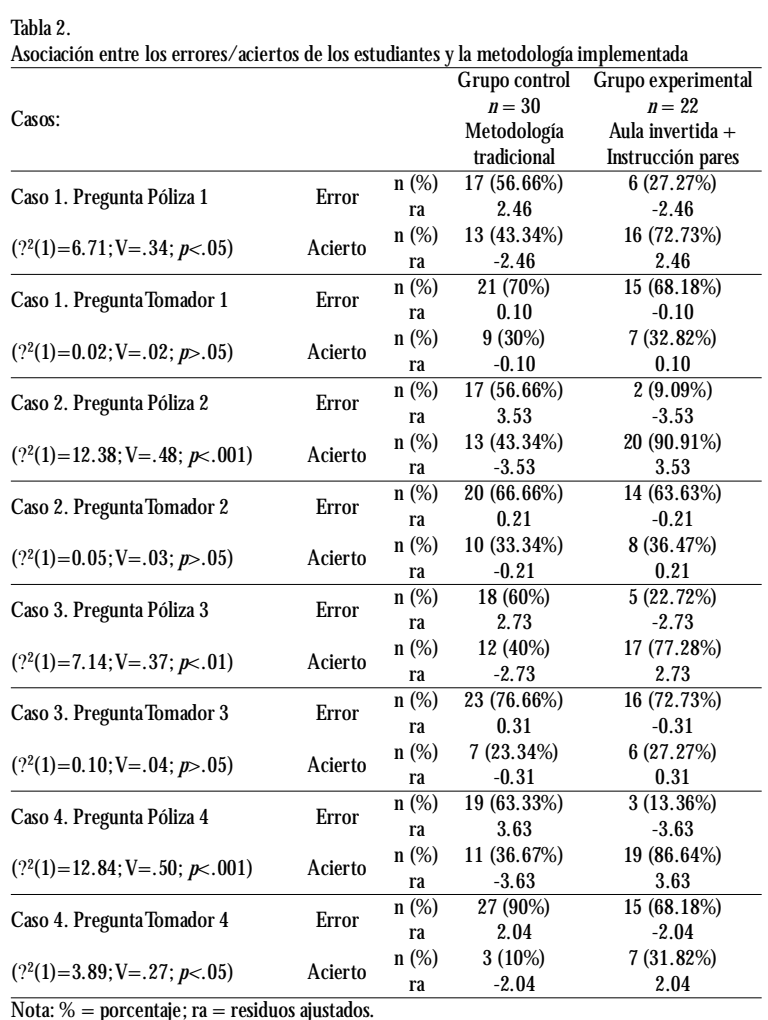

\section{Discusión}

Es bien sabido que las metodologías innovadoras centradas en el alumnado reportan beneficios sobre su aprendizaje y rendimiento académico (Balta et al., 2017; Hinojo-Lucena et al. , 2019). N umerosos estudios en diferentescontextos (e.g., Hernández-Campos\& M urilloQ uirós, 2019; Recio-Moreno et al., 2021; Sola et al., 2019) han demostrado de forma independiente como el aula invertida o la instrucción por pares tienen un efecto significativo sobreel aprendizaje. Sin embargo, a pesar de que por su características son dos metodologías fácilmente hibridables (Balta et al. , 2017; H inojo-Lucena et al., 2019), ninguna investigación hasta la fecha y para el conocimiento de los autores, ha examinado el efecto que podría tener combinar ambas metodologías en estudiantes universitarios (Zainuddin \& Halili, 2016). Con la intención de expandir la literatura científica, el objetivo del presente estudio fue evaluar el efecto de la combinación de estas metodologías sobre las calificaciones académicas obtenidas por los estudiantes universitarios en una asignatura de grado en Ciencias de laActividad Física y del Deporte. 
En línea con la hipótesis planteada y con estudios previos (e.g., Hinojo et al., 2019; Lasry et al., 2008), el alumnado del grupo experimental que recibió los contenidos de la asignatura a través de la combinación metodológica de aula invertida e instrucción entre pa res durante tres sesiones, obtuvo un mayor porcentaje de acierto en las respuestas del examen en compara ción con el grupo control. Particularmente, se observó una asociación positiva y significativa en cinco de las ocho preguntas entre esta combinación metodológica y responder correctamente. Los resultados encontrados pueden considerarse prometedores debido a que en solo tres sesiones destinadas a la utilización de estas metodologías se ha apreciado una mejora en las califica ciones académicas del alumnado universitario. Sin embargo, debido a la ausencia de estudios en la literatura que hayan evaluado los efectos conjuntos de la combina ción metodológica de aula invertida e instrucción entre pares en las cal ificaciones del alumnado, no ha sido posible comparar los resultados del presente estudio con trabajos previos. No obstante, los resultados obtenidos en este trabajo están en línea de otras investigaciones realizadas en diferentes disciplinas en el contexto universitario en las que se apreciaron mejores calificaciones académicas en el al umnado que recibió los contenidos con metodologías más activas como el aula invertida (Chen et al. , 2018; H ew \& Lo 2018; Hinojo-Lucena et al., 2019; Sola et al., 2019) o la instrucción entre pares(Baltaet al., 2017; Hernández-Campos\& MurilloQ uirós, 2019; Vickrey et al. , 2015). Por ejemplo, en un estudio realizado en estudiantes universitarios españoles en la especial idad de Educación Física en el G rado de Primaria se observó también una asociación positiva y significativa entre la metodología de aula invertida y las calificaciones obtenidas en una asignatura de dicho grado (Hinojo-Lucena, Mingorance-Estrada, Trujillo, Aznar-Díaz \& Cáceres, 2018).

Estos resultados podrían explicarse atendiendo a diversas razones. Por un lado, parece que la hibridación de estas dos metodologías, debido a sus características que fomentan la participación y la comprensión (Balta et al., 2017; Hinojo-Lucena et al., 2019), podría ayudar a que el alumnado universitario pase de tener un papel pasivo en el aula (i.e., un mero oyente) con la instrucción directa, a un papel mucho más activo y protagonista. Este rol de responsabilidad sobre su aprendizaje, asu vez, podría promover la reflexión, la cooperación y la comprensión de los estudiantes, dado que les obliga a interactuar y trabajar dentro y fuera del aula para alcanzar los objetivos (Arráez et al., 2018). Todo ello, podría explicar que el alumnado del grupo experimental del presente estudio, en comparación con el del grupo control, haya reportado mejores calificaciones, las cuales, parecen haber estado desencadenadas por un aprendizaje más profundo y significativo del contenido. No obstante, esta experiencia debe considerarse como una intervención piloto y son necesarias más investigaciones que analicen experimentalmente el efecto de hibridar las metodologías de aula invertida e instrucción entre pares con diseños más prolongados en el tiempo.

Por otro lado, en línea con los supuestos de la teoría de la autodeterminación (TAD) (Ryan \& Deci, 2017), estas metodologías pueden favorecer una motivación más autodeterminada del al umnado a través de la satisfacción de las necesidades psicológicas básicas de autonomía, competencia y relaciones sociales (Zainuddin \& Perera, 2019). Más precisamente, esta combinación metodológica puede satiffacer la autonomía de los estudiantes al permitirles aprender a su propio ritmo, pudiendo proporcionan flexibilidad en la fecha de entrega de las actividades y oportunidades de elección entre las distintas tareas a realizar (Persky \& M cLaughlin, 2017). Igualmente, el aula invertida y la instrucción entre pares se caracterizan por incluir autoevaluaciones que permiten a los estudiantes identificar sus fortal ezas y debilidades (López-Pastor et al., 2020), valorar su progreso del aprendizaje hacia el logro de los resultados esperados (López-Pastor et al., 2020), favorecer su satisfacción de competencia y satisfacer las relaciones sociales del alumnado al requerir constantes discusiones activas con diferentes compañeros durante las sesiones (Bermejo-Díaz etal., 2021; Persky \& M cLaughlin, 2017). De esta manera, parece que la satisfacción de las tres necesidades psicológicas básicas y, por ende, la mayor motivación autodeterminada de los estudiantes (Ryan \& Deci, 2017), podría ayudar a explicar el incremento en las tasas de acier to obtenidas en las respuestas del alumnado que recibió los contenidos a través de metodologías activas (Richardson, Abraham $\&$ Bond, 2012). Paralelamente, existe un reciente cuerpo de investigación basada la TAD ÿp(Fernández-Espínola, Almagro \& Tamayo-Fajardo, 2020; González-Cutre, Romero-Elías, Jiménez-Loaisa\& Beltrán-Carrillo, 2020; González-Cutre y Sicilia, 2019) que ha demostrado experimentalmente cómo la satisfacción de novedad (i.e., experimentar algo nuevo 0 inusual) puede favorecer la motivación y otras consecuencias positivas. De este modo, esto podría explicar que haber recibido una metodología diferente a la que normalmente están acostumbrados los alumnos de grado (Arráez et al. 2018), 